\title{
Recent Change in Management Pattern of Meconium lleus at Dhaka Shishu Hospital
}

\author{
Kazi Md. Noor-ul Ferdous ${ }^{1 *}$ \\ Md. Samiul Hasan ${ }^{1}$ \\ Ashfaq $\mathrm{Nabi}^{1}$ \\ Md. Arman Hossain ${ }^{1}$ \\ M. Kabirul Islam ${ }^{1}$ \\ Tahmina Banu² \\ ${ }^{1}$ Division of Pediatric Surgery \\ Bangladesh Institute of Child Health (BICH) \& \\ Dhaka Shishu Hospital \\ Dhaka, Bangladesh. \\ ${ }^{2}$ Department of Pediatric Surgery \\ Chittagong Research Institute for Children Sugary (CRICS) \\ Chittagong, Bangladesh.
}

\section{${ }^{*}$ Correspondence to:}

\section{Dr. Kazi Md. Noor-Ul Ferdous}

Assistant Professor

Dhaka Shishu Hospital

Dhaka, Bangladesh.

Mobile : +8801711322487

E-mail:kmnferdous@gmail.com

www.banglajol.info/index.php/CMOSHMCJ

\begin{abstract}
Background : Meconium Ileus (MI) accounting for 9-33\% of neonatal intestinal obstruction and one of the common cause of intestinal obstruction in neonate. Near about $50 \%$ can be present with various complications. Options for surgical management of MI include resection of dilated ileum with primary anastomosis, Bishop-Koop ileostomy, Santulli procedure or Mikulicz procedure and T tube ileostomy. In our center, before January 2015, mostly Mikulicz and few BishopKoop procedures were done for MI. But, the complications were high. From 2015, we started to perform T tube ileostomy and Bishop-Koop procedure in simple MI. In complicated cases Mikulicz and Bishop-Koop procedure were done and the scenario changed. This study was done to compare the outcome of MI surgery before and after January 2015. Methods: This was a retrospective study done in our center during January 2011 to December, 2016 (5 years). All cases were divided into two groups (Group-A: January 2011 to December 2014 and in Group-B: January 2015 to December 2016). Demographic data and surgical outcomes were compared. Results: Total 216 cases were included, in Group-A: 123 patients and others were in GroupB. Simple MI were $49 \%$ in Group-A and $56 \%$ were in Group-B. Complicated MI presented with small gut volvulus (43.3\%) gangrene of loaded ileum $(32.2 \%)$ perforation with peritonitis $(37.8 \%)$ intestinal atresia with adhesions (16.3\%) and meconium pseudocysts in 12 cases (16\%). 11 patients had more than one complication. All the postoperative complications were found significantly high in Group-A except anastomotic leakage. Mortality was also significantly high in Group-A. Conclusion: Morbidity and mortality were significantly reduced with increasing practice of $\mathrm{T}$ tube ileostomy and Bishop-Koop procedure in simple and complicated Meconium ileus.
\end{abstract}

Key words: Meconium ileus; Bishop Koop ileostomy; T tube ileostomy; Mikulicz procedure.

\section{INTRODUCTION}

Meconium Ileus (MI) accounting for $9-33 \%$ of neonatal intestinal obstruction and one of the common cause of intestinal obstruction in neonate. Here obstruction occur secondary to the intraluminal accumulation of inspissated and desiccated meconium within the lumen ${ }^{1,2}$. Recent studies demonstrate that it can occur frequently without association of cystic fibrosis. Though, previously it was considered to be closely associated with cystic fibrosis. The exact pathogenesis of MI in the absence of cystic fibrosis is yet to be known, a spectrum of genetic and pathological abnormalities may play a role ${ }^{3,4}$. There are two types of meconium ileus, simple and complicated. In simple MI, thickened sticky meconium accumulates within the dilated thickened walled mid ileum and causes obstruction. About $40-50 \%$ of patients can be presented with various form e.g. volvulus, atresia, necrosis perforation, or meconium pseudocyst, termed as complicated $\mathrm{MI}^{1,2}$. Simple 
MI can be treated with therapeutic contrast enemas with watersoluble and hyper or iso-osmolar contrast ${ }^{5}$. There are some criteria, which should be followed before performing the conservative procedure to avoid the complications like perforation, necrotizing enterocolitis, shock and occasional death $^{5,6}$. Success rate of non-operative management of simple MI is about 60 to $70 \%$ of newborns and current short-term operative survival rates of 70 to $100 \%$ are reported ${ }^{7}$. Options for surgical management of MI include resection of dilated ileum with primary anastomosis, Bishop-Koop ileostomy, Santulli procedure or Mikulicz procedure, T tube ileostomy ${ }^{1,2,8,9}$

Bishop-Koop procedure, Santulli procedure and Mikulicz procedure are extensive operation, associated with gut reduction. Though Bishop-Koop procedure allows function of distal bowel and stoma output is minimum, Mikulicz procedure associated with high output stoma, failure to thrive, sepsis, recurrent hospital admission and higher mortality. A second surgery also required to close the stoma in those procedures ${ }^{10,11}$. T-tube ileostomy includes enterotomy, evacuation of thick meconium and placement of T-tube for post-operative irrigation ${ }^{8,9}$. This procedure do not require gut resection and there is no intraperitoneal anastomosis. After extraction of T-tube the wound heal spontaneously and second operation to close the stoma is not required ${ }^{11}$.

It is not possible for us to manage simple MI conservatively as we do not have fluoroscopy facilities. Before January 2015, we used to perform Mikulicz procedures in most of the cases of simple and complicated MI. Only few cases of simple MI were treated with Bishop-Koop procedure. From January 2015, we started to perform $\mathrm{T}$ tube ileostomy and Bishop-Koop procedure in simple MI. In complicated cases we are doing Mikulicz and Bishop-Koop procedure.

This retrospective study was done to compare these surgical procedures used in the treatment of MI and to see their efficacy regarding survival and complications before and after 2015 .

\section{MATERIALS AND METHODS}

This retrospective study was done in all cases of MI admitted to the Division of Pediatric Surgery, Bangladesh Institute of Child Health (BICH) \& Dhaka Shishu (Children) Hospital, Dhaka, Bangladesh during the past 5 years (January 2011 to December 2016). The medical records of cases of MI were studied. We excluded the cases with incomplete data. After initial resuscitation patients were taken to operation room. After confirmation of $\mathrm{MI}$ and its type at laparotomy different operative procedures (Mikulicz/ Bishop Koop/T tube ileostomy) performed depending on patient's condition and surgeons' choice. All the study cases were divided into two groups. In Group -A, patients whom operated before January 2015 and in Group-B, patients whom operated after that. Surgical outcomes were compared between two groups.

\section{T-Tube Ileostomy procedure}

After confirmation of diagnosis simple MI at laparotomy, an enterotomy was made on antimesenteric border of ileum, 3-4 $\mathrm{cm}$ proximal to distal narrow segment. Thick meconium and pellets were evacuated through the enterotomy with minimal bowel handling. A 12/14 Fr T-tube was inserted through the enterotomy and was secured with double purse string suture with catgut. Irrigation was given with saline and $2 \% \mathrm{~N}$-acetyl cysteine $($ Ratio $=9: 1)$. The T-tube was brought out through a stab incision in the right iliac fossa and enterostomy was secured to the anterior abdominal wall for free drainage. $\mathrm{T}$ tube was irrigated two times with $10 \mathrm{ml}$ of saline and $2 \% \mathrm{~N}$ acetyl cysteine $(9: 1)$ starting from $1^{\text {st }}$ postoperative day until spontaneous bowel movement establishes. Then oral feeding started and $\mathrm{T}$ tube was removed.

Statistical significance is determined by using the SPSS software, version 22. Associations of continuous data were assessed using student t- test. Associations of categorical data were assessed using Chi-square test and Fisher's exact test. For both test, $\mathrm{p}<0.05$ was considered significant.

\section{RESULTS}

The incidence of meconium ileus was 56.4 per 1000 neonatal surgical cases at the center. During the study period total 224 neonates were admitted with MI. Eight patients were excluded for inadequate data. Age at presentation was range from 2 days to 6 days. Male and female were 111 and 105 in number and the ratio was $1.05: 1$. Preterm represented $11.11 \%$ of all cases. One hundred twenty three patients included in Group-A, whom were operated before January 2015 and 93 were in Group-B, whom were operated after January 2015 [Table 1].

Table 1: Age, Sex \& weight distribution among two groups $(n=216)$.

\begin{tabular}{|c|c|c|c|c|}
\hline \multicolumn{2}{|c|}{ Variables } & Group-A $(n=123)$ & Group-B $(n=93)$ & p \\
\hline \multicolumn{2}{|c|}{ Mean Age (days) } & 3.29 & 3.16 & \\
\hline \multicolumn{2}{|c|}{ Premature } & 15 & 9 & \\
\hline \multirow[t]{2}{*}{ Sex } & Male & 68 & 43 & NS \\
\hline & Female & 55 & 50 & \\
\hline \multicolumn{2}{|c|}{ Mean Weight $(\mathrm{kg})$} & 2.43 & 2.54 & \\
\hline
\end{tabular}

Among the studied cases, simple MI were $49 \%$ in Group-A and $56 \%$ were in Group-B (Figure-1). There was no significant difference between two groups.

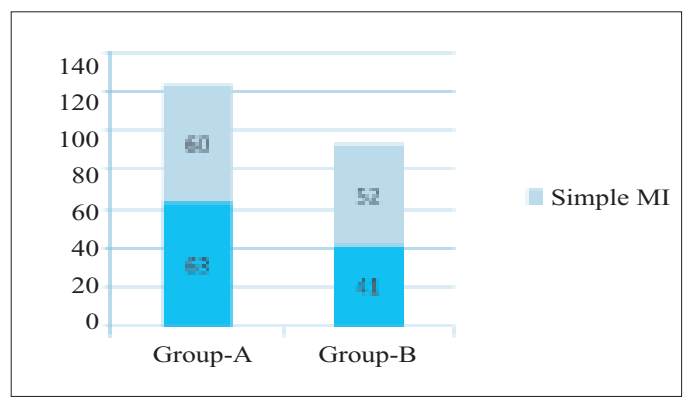

Figure 1: Types of Meconium ileus in both groups. 
In complicated MI, the complications were small gut volvulus (43.3\%) gangrene of loaded ileum $(32.2 \%)$ perforation with peritonitis $(37.8 \%)$ intestinal atresia with adhesions (16.3\%) and meconium pseudocysts in 12 cases (16\%). 11 patients had more than one complication.

Table 2 : Distribution of types of operations.

\begin{tabular}{lcccc} 
Operation & \multicolumn{2}{c}{ Group-A(n=123) } & \multicolumn{2}{c}{ Group-B(n=93) } \\
& Simple MI & Complicated MI & Simple MI & Complicated MI \\
Miculicz & 32 & 49 & - & 26 \\
Bishop-Koop & 28 & 14 & 10 & 15 \\
T-Tube & -- & -- & 42 & -- \\
\hline
\end{tabular}

Mortality was high in Group-A. In Group-A, 53 neonates died out of 123 and 26 cases out of 93 in Group-B. This difference was significant $(\mathrm{p}=.023)$. Figure 2 shows mortality pattern.

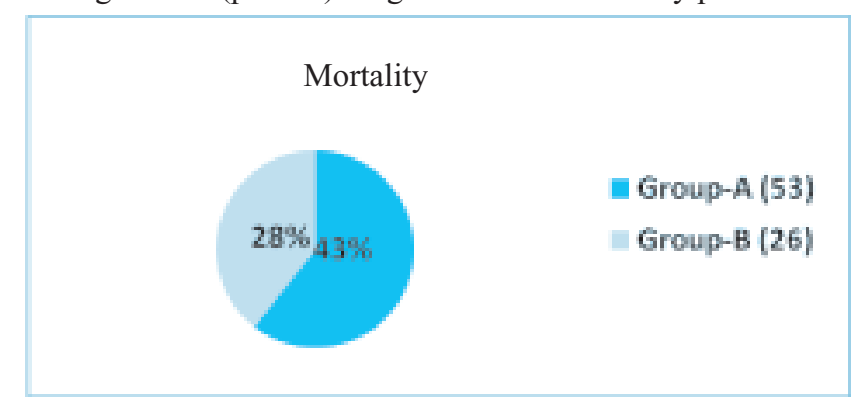

Figure 2 : Mortality pattern among two Groups.

Post-operative complications were anastomotic leakage, peristomal skin excoriation, high stomal output and sepsis. All the complications were found significantly high in Group-A except anastomotic leakage. In Group-A, all 11 anastomotic leakage occurred in Bishop-Koop procedure and in Group-B, 8 in Bishop-Koop procedure and 2 in T tube ileostomy [Table 3].

Table 3 : Different complications among the groups.

\begin{tabular}{lccc} 
Variable & Group-A (n=123) & Group-B (n=93) & p \\
Leakage & $11(9 \%)$ & $10(11 \%)$ & NS \\
Excoriation & $54(44 \%)$ & $17(18 \%)$ & .000 \\
Sepsis & $62(50 \%)$ & $29(31 \%)$ & .005 \\
High stomal output & $59(48 \%)$ & $21(23 \%)$ & .000 \\
Mortality & $53(43 \%)$ & $26(28 \%)$ & .023 \\
\hline
\end{tabular}

In Group-A, after survival of 70 cases all needed second operation for stoma closure. Whereas, in Group-B, after survival of 67 cases only 25 needed second operation for stoma closure.

\section{DISCUSSION}

In early decayed of twentieth century, Meconium ileus was considered to be a fatal condition in neonatal surgery. The scenario became changed at 1948, when Hiatt and Wilson introduced enterotomy and saline irrigation for clearance of the obstructing pellets. With the improvement of neonatal surgical care over past decades survival is now approaching 95 to $100 \%{ }^{1,2}$. But, outcome of meconium ileus still not up to the mark in our setup for some limitations.
There are no significant different in demographic data and regarding the types of MI between the study groups. The predominant complications of MI were volvulus, gangrene, perforation and atresia seen in both groups. Delphin et al, Rescorla et al and Karimi et al reported similar findings ${ }^{7,8,12}$.

Significant differences were found between the types surgical procedures in both groups in this study. In Group-A, Mikulicz ileostomy were done in most of the cases. In presence of contamination our surgeons preferred Mikulicz procedure as it requires less operation time and no intraperitoneal suture line. Hence, it reduces duration of anesthesia in these sick patients ${ }^{13}$.

But, complications like high output fistula, skin excoriation and sepsis were significantly higher in Group-A as, Mikulicz procedure were predominant in those. Intra peritoneal leakage was found more in Bishop Koop procedure followed by $\mathrm{T}$ tube ileostomy. Delpin et al and Karimi et al mentioned higher risk of leakage in Bishop Koop procedure ${ }^{8,12}$. In Bishop Koop procedure intestinal contents passes into distal bowel, thus more fluid \& nutrients are absorbed. So, high output fistula, skin excoriation and sepsis are less ${ }^{14}$. One major disadvantage of Mikulicz and Bishop Koop procedure is, these procedures required another operation to close stoma ${ }^{1,2,12,14}$.

In our study, $\mathrm{T}$ tube ileostomy only performed in simple cases in Group-B. Here, intra peritoneal leakages were few and complications were negligible. After first description by Harberge et al, several author described $\mathrm{T}$ tube ileostomy as a safe and effective procedure for simple $\mathrm{MI}^{8,10,11}$. Samiul $\mathrm{H}$ et al also found results of $\mathrm{T}$ tube ileostomy is better than BishopKoop procedure in simple $\mathrm{MI}^{15}$.

There was significant difference in need of second operation for stoma closure between two groups. This is because of introduction of $\mathrm{T}$ tube ileostomy in simple MI. This advantage also mentioned by other authors ${ }^{10,11,15}$.

The mortality of MI was significantly reduced in Group-B. Introduction of $\mathrm{T}$ tube ileostomy and increasing practice of Bishop-Koop procedure contributed to this improved outcome. Several studies shown that mortality was low in T-tube ileostomy and Bishop-Koop procedure ${ }^{9-14}$. Mortality was significantly higher in Group-A, whom Mickulicz procedure done mostly. It was very difficult to manage these patients without neonatal intensive care support and total parenteral nutrition.

\section{CONCLUSION}

Morbidity and mortality were significantly reduced with increasing practice of $\mathrm{T}$ tube ileostomy and Bishop-Koop procedure in simple and complicated Meconium ileus. Mikulicz procedure should be reserved for highly contaminated complicated cases and early stoma closure should be considered.

\section{DISCLOSURE}

All the authors declared no competing interest. 


\section{REFERENCES}

1. Michael G. Caty, Mauricio A. Escobar Jr. Meconium ileus, in: Ashcraft's Pediatric Surgery $6^{\text {th }}$ Ed. George W, Holcomb III, Patric J, Murphy, Daniel J. Ostlie. 2014; .32:439- 446.

2. Zeigler MM, Meconium ileus, in: Pediatric Surgery $7^{\text {th }}$ Ed., Coran A.G, Adzick N.S, Krummel T.M, Laberge J, Shamberger R.C, Caldamone A.A. Elsevier, Philadelphia. 2012; 1073-1083.

3. Fakhoury K, Durie P.R., Levison H, Canny G.J. Meconium ileus in the absence of cystic fibrosis, Archives of Disease in Childhood. 1992; 67:1204-1206.

4. Gorter R.R, Karimi A, Sleeboom C, Kneepkens C.M, Heij H.A. Clinical and genetic characteristics of meconium ileus in newborns with and without cystic fibrosis. J Pediatr Gastroenterol. 2010; 50(5):569-570.

5. Noblett H.R. Treatment of Uncomplicated Meconium Ileus by Gastrografin Enema: A Preliminary Report. Journal of Pediatrics Surgery. 1969; 4(2):190-197.

6. Copeland D. R, St. Peter S.D, Sharp S.W, Islam S, Cuenca A, Tolleson J.S. Diminishing role of contrast enema in simple meconium ileus. Journal of Pediatric Surgery. 2009; 44: 2130-2132.

7. Delpin C.A, Czyrko C, Ziegler M.M, Scanlin T.F, Bishop H.C. Management and survival of Meconium Ileus: A 30 years review. Annals of Surgery. 1992;215(2):179-85. Doi:10.1097/00000658-1992020000-00014.

8. Rescorla F.J, Grosfeld J.L. Contemporary management of meconium ileus. World J Surg. 1993;17(3):318-325.

9. Harberg F.J, Senekjian E.K, PoKorny W.J. Treatment of uncomplicated meconium ileus via T-tube ileostomy. Journal of Pediatric Surgery. 1981;16: 61-63.

10. Bhattacharyaya S, Basu K.S, Samanta N. Proximal bowel T-tube drainage and local instillation of N-acetyl cysteine. Journal of Indian Association of Pediatric Surgery. 2005;10(1):37-40.

11. Mak G.Z, Harberg F.J, Hiatt P, Deaton A, Calhoon R, Brandt M.L. T-tube ileostomy for meconium ileus: Four decades of experience. Journal of Pediatric Surgery. 2000; 35(2):349-352.

12. Karimi A, Gorter R.R, Sleeboom C, Kneepkens C.M.F, Heij H.A. Issues in the management of simple and complex meconium ileus. Pediatric Surgery International. 2011; 27:963-968.

13. Haithem H. A. Meconium ileus. A study and comparison between common operative procedures Performed in Basrah. Basrah Journal of Surgery. 2016; 22, 84-90.

14. Bishop H.C, Koop C.E. Management of meconium ileus: Resection, Roux-en-Y anastomosis and ileostomy irrigation with pancreatic enzymes. Annals of Surgery. 1957; 145(3): 410-414.

15. Hasan M.S, Mitul A.R, Karim S, Noor-ul Ferdous K.M, Kabirul Islam M. Comparison of T tube ileostomy and Bishop-Koop ileostomy for the management of uncomplicated meconium ileus. J Neonatal Surg. 2017;6:56. Doi: 10.21699/jns.v6i3.617. 